\title{
Características y mortalidad en pacientes mexicanos con COVID-19 y ventillación mecánica
}

Pablo Álvarez-Maldonado, ${ }^{1 *}$ Grisel Hernández-Ríos, ${ }^{1}$ Julio C. Ambríz-Mondragón, ${ }^{1}$ Julián A. Gordillo-Mena, ${ }^{1}$ Diana F. Morales-Serrano, ${ }^{1}$ Arturo Reding-Bernal ${ }^{2}$ y Alejandro Hernández-Solis ${ }^{1}$

${ }^{1}$ Servicio de Neumología y Cirugía de Tórax; 'División de Investigación. Hospital General de México "Dr. Eduardo Liceaga", Ciudad de México, México

\section{Resumen}

Introducción: La mortalidad por COVID-19 en quienes requieren ventilación mecánica se desconoce en la población mexicana. Objetivo: Describir las características de pacientes mexicanos con COVID-19 que requirieron ventilación mecánica. Métodos: Estudio de cohorte observacional en una unidad de terapia intensiva, del 25 de marzo al 17 de julio de 2020. Los datos se obtuvieron de una base de datos prospectiva y de registros clínicos electrónicos; fueron analizados con $\chi^{2}$, prueba exacta de Fisher 0 prueba U de Mann-Whitney. Resultados: Cien pacientes recibieron ventilación mecánica, la edad media fue de 56 años, $31 \%$ era del sexo femenino y $97 \%$, latinoamericano. Las comorbilidades más comunes fueron obesidad (36 \%), diabetes (26 \%), hipertensión (20\%) y enfermedad renal crónica o renal terminal (10\%). Al término del análisis, 11 pacientes permanecían en la UCI, 31 egresaron vivos y 58 (65.2\%) fallecieron; los sobrevivientes fueron más jóvenes, con menor puntuación en las escalas de gravedad y disfunción orgánica, menores niveles de proteína C reactiva al ingreso a la UCl, menor propensión a hemodiálisis y necesidad de vasopresores y con mayor estancia hospitalaria y en la UCl. Conclusiones: Este estudio agrega información sobre la presentación y resultados de pacientes con ventilación mecánica infectados con SARS-CoV-2.

PALABRAS CLAVE: COVID-19. SARS-CoV-2. Ventilación mecánica. México. Latinoamérica.

\section{Characteristics and mortality of Mexican patients with COVID-19 and mechanical ventilation}

\section{Abstract}

Introduction: COVID-19 associated mortality in patients who require mechanical ventilation is unknown in the Mexican population. Objective: To describe the characteristics of Mexican patients with COVID-19 who required mechanical ventilation. Methods: Observational cohort study carried out in an intensive care unit from March 25 to July 17, 2020. Data were obtained from a prospective database and electronic medical records, and were analyzed with the chi-square test, Fisher's exact test or Mann-Whitney's U-test. Results: One hundred patients required mechanical ventilation; median age was 56 years, $31 \%$ were females and $97 \%$ were Latin American. Most common comorbidities were obesity (36 \%), diabetes (26\%), hypertension (20\%), and chronic or end-stage kidney disease (10\%). At the end of the analysis, 11 patients remained in the ICU, 31 had been discharged alive and 58 (65.2\%) died; survivors were younger, had lower scores on severity and organ dysfunction scales, lower levels of C-reactive protein at ICU admission, were less likely to receive hemodialysis and vasopressors, and had longer hospital and ICU stays. Conclusions: This study adds information on the presentation and results of SARS-CoV2-infected patients who require mechanical ventilation.

KEY WORDS: COVID-19. SARS-CoV-2. Mechanical ventilation. Mexico. Latin America.

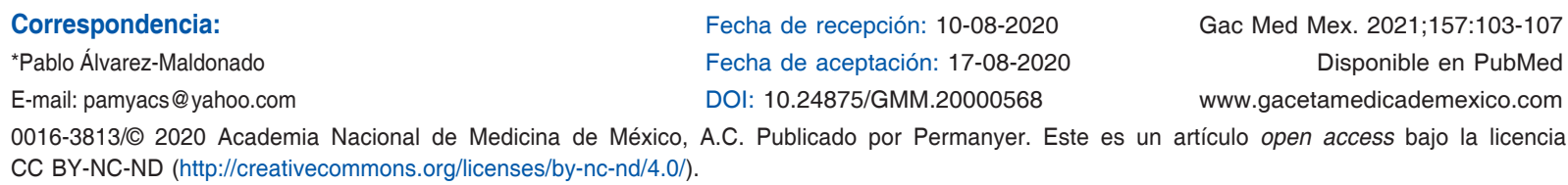
CC BY-NC-ND (http://creativecommons.org/licenses/by-nc-nd/4.0/).

Gac Med Mex. 2021;157:103-107

Disponible en PubMed

www.gacetamedicademexico.com 


\section{Introducción}

La enfermedad por el coronavirus 2019 (COVID-19) exige cuidados intensivos en 3 a $5 \%$ de los enfermos. Actualmente, la pandemia está en su apogeo en muchos países, ${ }^{1}$ con una mortalidad que oscila entre 35.7 y $96.8 \%$ en quienes requieren ventilación mecánica. ${ }^{2-4}$

El Hospital General de México "Dr. Eduardo Liceaga" es un centro de referencia con 1131 camas en la Ciudad de México, el cual proporciona atención a 800000 habitantes. Dado que hasta el momento no hay series publicadas sobre la mortalidad de pacientes con COVID-19 que requieren ventilación mecánica en México ni en países latinoamericanos, describimos nuestros resultados después de más de tres meses de actividad clínica durante la pandemia (del 25 de marzo al 17 de julio de 2020).

\section{Métodos}

Estudio de cohorte observacional de pacientes con infección por SARS-CoV-2 (severe acute respiratory syndrome coronavirus 2), confirmada por reacción en cadena de la polimerasa en tiempo real, que desarroIlaron insuficiencia respiratoria aguda y requirieron ventilación mecánica. La Unidad de Cuidados Intensivos (UCl) Respiratorios del Hospital General de México "Dr. Eduardo Liceaga" fue la primera área de cuidados críticos que recibió pacientes en el hospital durante la pandemia, tiene 12 camas y al principio fue atendida por médicos intensivistas en horario diurno, apoyados por residentes de neumología de segundo y tercer año. Un mes después de decretada la pandemia en México, al equipo se agregaron un intensivista y dos neumólogos. No hubo escasez crítica de medicamentos, ventiladores mecánicos, máquinas de hemodiálisis intermitente ni equipos de protección personal. La falta de intercambiadores de calor y humedad fue subsanada por los familiares de los pacientes en un tercio de las ocasiones. El sistema de salud de México no alcanzó su máxima capacidad durante el periodo de estudio.

Los datos para el análisis se extrajeron de la base prospectiva de datos local y directamente de los registros clínicos electrónicos; las variables categóricas fueron analizadas con chi cuadrada o prueba exacta de Fisher y las variables continuas con $U$ de Mann-Whitney, con un valor de $p<0.05$, considerado estadísticamente significativo (Stata ${ }^{\circledR}$, StataCorp LLC,
College Estación, TX, Estados Unidos). Los comités de investigación y ética aprobaron este estudio. Se mantuvo el anonimato de los pacientes.

\section{Resultados}

De 104 pacientes con COVID-19 ingresados en el periodo de estudio, 35 (33.7 \%) fueron transferidos vivos de la $\mathrm{UCl}$ y 58 murieron en la $\mathrm{UCl}$ (tasa de mortalidad en la UCI de $62.4 \%$ ), ocho muertes adicionales ocurrieron entre los pacientes transferidos a piso de hospitalización, uno de ellos que tenía una orden de no intubar murió poco después del alta de la UCl; 24 pacientes fueron dados de alta vivos del hospital y tres aún permanecían en el hospital.

Cien pacientes fueron sometidos a ventilación mecánica, la mediana de edad fue de 56 años (rango intercuartílico, 49-66), 31 eran mujeres (31\%) y 97 eran latinoamericanos (97\%). La obesidad fue la afección comórbida más común (36\%), seguida de diabetes (26\%), hipertensión (20\%) y enfermedad renal crónica o enfermedad renal en etapa terminal $(10 \%)$. Treinta y un pacientes fueron dados de alta vivos de la UCl (Figura 1). De los 58 pacientes que murieron (la tasa de mortalidad de pacientes en ventilación mecánica en la $\mathrm{UCl}$ fue de $65.2 \%$ ), cuatro tenían indicación de limitación o retirada de tratamiento, los otros 54 recibieron soporte vital total. Once pacientes permanecieron en la $\mathrm{UCl}$ y al momento de este informe todavía recibían ventilación mecánica. Los datos sociodemográficos, características clínicas basales, intervenciones y los resultados se resumen en la Tabla 1.

\section{Discusión}

El primer caso de COVID-19 en México se detectó el 27 de febrero de 2020. Aunque más adelante datos epidemiológicos mostraron similitudes entre los casos de México y China, se observó reducción en el número de casos confirmados y mayor tasa de mortalidad en México en el mismo periodo de la curva epidémica en comparación con China. ${ }^{5}$ Este hecho generó preocupaciones sobre si factores regionales, incluida la prestación de cuidados críticos, estaban influyendo en los resultados. Nuestra experiencia inicial con esta cohorte de pacientes con COVID-19, con ventilación mecánica y atendidos en el sistema de salud pública, demostró una tasa de mortalidad situada en medio de las reportadas en la literatura hasta la fecha. A pesar de ser considerablemente 


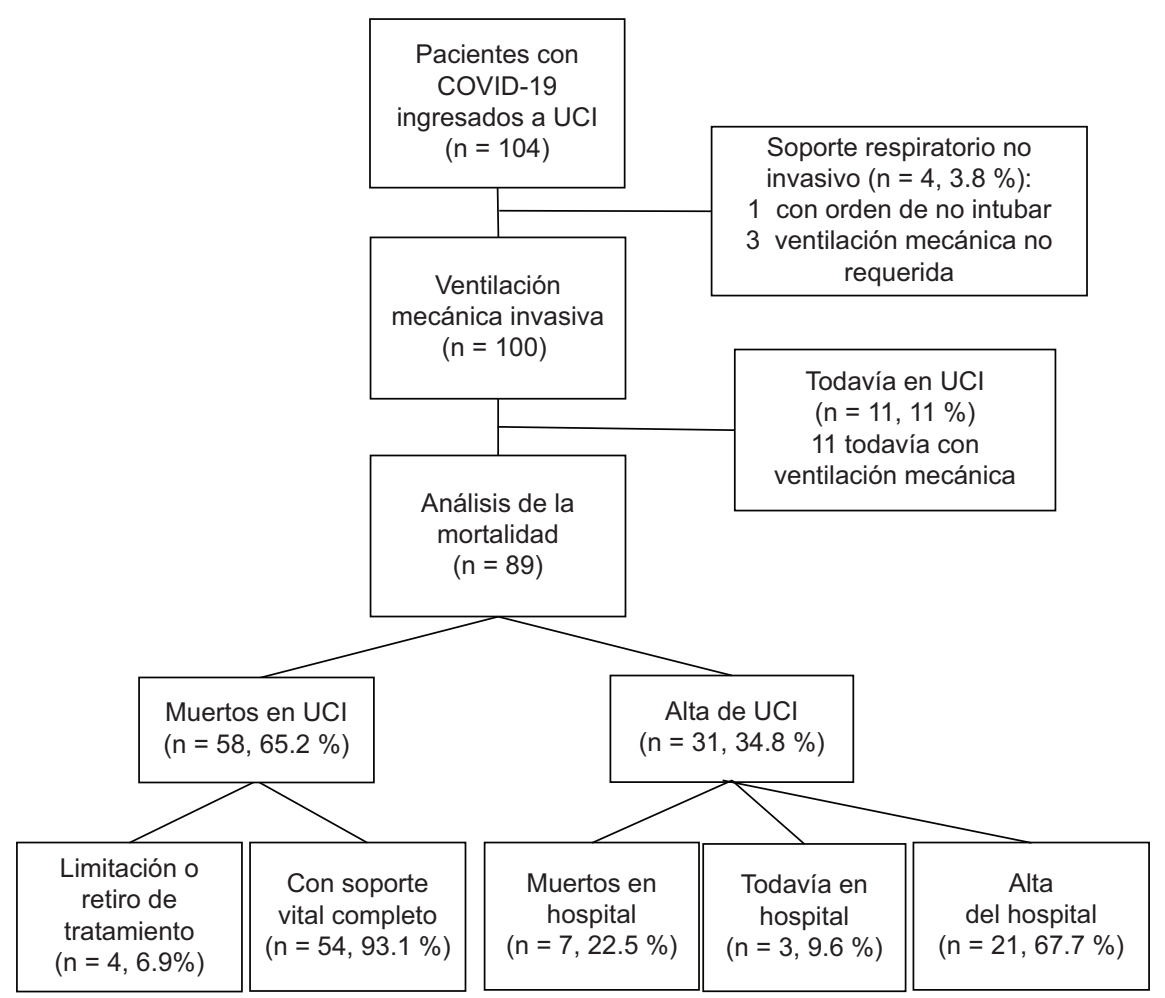

Figura 1. Diagrama de flujo de pacientes con COVID-19 que ingresaron a la unidad de cuidados intensivos (UCl) y recibieron ventilación mecánica invasiva.

alta, Arentz et al. ${ }^{6}$ (Estados Unidos), Richardson et al. ${ }^{3}$ (Estados Unidos.) y Zhou et al. ${ }^{4}$ (China) informaron en sus primeras experiencias tasas de mortalidad de 85,88 y $96.8 \%$, respectivamente. Por el contrario, Zangrillo et al. ${ }^{7}$ (Italia), Ferrando et al. ${ }^{8}$ (España) y Auld et al. ${ }^{2}$ (Estados Unidos) indicaron tasas de mortalidad de $42.5,37.5$ y $35.7 \%$.

Los pacientes que sobrevivieron en la $\mathrm{UCl}$ fueron significativamente más jóvenes, no hubo diferencias en la supervivencia entre sexos ni cuando se compararon los grupos étnicos; sin embargo, solo tres pacientes blancos fueron incluidos en la cohorte.

Los datos de los primeros 12656 pacientes infectados de SARS-CoV-2 en México, emitidos por la Secretaría de Salud, mostraron predominio de obesidad, diabetes e hipertensión arterial entre quienes fallecieron..$^{5}$ También Kammar García et al. ${ }^{9}$ encontraron mayor riesgo de eventos adversos y muerte en pacientes mexicanos con COVID-19 cuando se combinaron estas tres comorbilidades. Aun así, en esta cohorte no encontramos diferencias estadísticamente significativas al comparar las comorbilidades entre los pacientes que sobrevivieron y los que murieron en la UCl.
La puntuación de las escalas SAPS-3 (Simplified Acute Physiology Score) y SOFA (Sequential Organ Failure Assessment), así como la proteína $C$ reactiva al ingreso a la UCI fueron significativamente más altas entre los que fallecieron, quienes, además, fueron más propensos a recibir terapia de reemplazo renal y vasopresores. En un centro COVID-19 en México se reportaron niveles significativamente más altos de proteína $\mathrm{C}$ reactiva respecto a otros biomarcadores inflamatorios en los pacientes ingresados a la $\mathrm{UCl}$ en comparación con quienes permanecieron en piso. ${ }^{10}$

Todos los pacientes que murieron tenían la forma grave del síndrome de insuficiencia respiratoria aguda $\left(\mathrm{PaO}_{2} /\right.$ $\mathrm{FiO}_{2}<100$ ) al ingreso a la UCl. Se encontraron estancias en UCl y hospital más prolongadas en quienes sobrevivieron en la $\mathrm{UCl}$. Un análisis reciente que incluyó a 103 pacientes con COVID-19 ventilados mecánicamente de $13 \mathrm{UCl}$ en Israel informó hallazgos similares respecto a la estadía en la $\mathrm{UCl}$ entre los supervivientes. ${ }^{11}$

Durante el periodo de atención clínica analizado, la escasez en la $\mathrm{UCl}$ se relacionó principalmente con la falta de personal médico especializado en todos sus turnos; sin embargo, una de las fortalezas fue que los ingresos a la $\mathrm{UCl}$ antes de la pandemia eran 
Tabla 1. Datos sociodemográficos, características clínicas basales, intervenciones y resultados de pacientes con COVID-19 y ventilación mecánica invasiva

\begin{tabular}{|c|c|c|c|c|c|c|c|}
\hline \multirow[t]{2}{*}{ Variables } & \multicolumn{2}{|c|}{$\begin{array}{l}\text { Todos los pacientes } \\
\qquad(\mathrm{n}=100)\end{array}$} & \multicolumn{2}{|c|}{$\begin{array}{l}\text { Sobrevivieron en } \\
\text { UCI }(n=31)\end{array}$} & \multicolumn{2}{|c|}{$\begin{array}{l}\text { Murieron en UCI } \\
\qquad(n=58)\end{array}$} & \multirow[t]{2}{*}{$\mathbf{p}^{*}$} \\
\hline & $\mathrm{n}$ & $\%$ & $n$ & $\%$ & $\mathbf{n}$ & $\%$ & \\
\hline Sexo femenino & 31 & 31 & 13 & 42 & 1526 & & $0.120^{\ddagger}$ \\
\hline $\begin{array}{l}\text { Tipo de población } \\
\text { Latinoamericana } \\
\text { Blanca }\end{array}$ & $\begin{array}{c}97 \\
3\end{array}$ & $\begin{array}{c}97 \\
3\end{array}$ & $\begin{array}{c}31 \\
0\end{array}$ & $\begin{array}{c}100 \\
0\end{array}$ & $\begin{array}{c}55 \\
3\end{array}$ & $\begin{array}{c}94.8 \\
5.2\end{array}$ & $0.549^{* *}$ \\
\hline Obesidad (índice de masa corporal $\geq 30$ ) & 36 & 36 & 8 & 25.8 & 25 & 43.1 & $0.107^{\ddagger}$ \\
\hline Diabetes & 26 & 26 & 6 & 19.4 & 17 & 29.3 & $0.307^{\ddagger}$ \\
\hline Hipertensión arterial & 20 & 20 & 2 & 6.5 & 12 & 20.7 & $0.125^{\star *}$ \\
\hline Enfermedad renal crónica/falla renal terminal & 10 & 10 & 3 & 9.7 & 6 & 10.3 & $1.000^{* *}$ \\
\hline $\begin{array}{l}\mathrm{PaO}_{2} / \mathrm{FiO}_{2} \\
\quad>100<200 \\
<100\end{array}$ & $\begin{array}{c}5 \\
95\end{array}$ & $\begin{array}{c}5 \\
95\end{array}$ & $\begin{array}{c}5 \\
26\end{array}$ & $\begin{array}{l}16.1 \\
83.9\end{array}$ & $\begin{array}{c}0 \\
58\end{array}$ & $\begin{array}{c}0 \\
100\end{array}$ & $0.004^{\star *}$ \\
\hline $\begin{array}{l}\text { Intervenciones en UCI } \\
\text { Azitromicina } \\
\text { Cloroquina/hidroxicloroquina } \\
\text { Tocilizumab } \\
\text { Plasma de donador convaleciente } \\
\text { Hemodiálisis intermitente } \\
\text { Cualquier vasopresor }\end{array}$ & $\begin{array}{c}88 \\
63 \\
15 \\
4 \\
19 \\
91\end{array}$ & $\begin{array}{c}88 \\
63 \\
15 \\
4 \\
19 \\
91\end{array}$ & $\begin{array}{c}27 \\
23 \\
7 \\
0 \\
2 \\
24\end{array}$ & $\begin{array}{c}87.1 \\
74.2 \\
22.6 \\
0 \\
6.5 \\
77.4\end{array}$ & $\begin{array}{c}49 \\
40 \\
8 \\
3 \\
15 \\
58\end{array}$ & $\begin{array}{c}84.4 \\
68.9 \\
13.8 \\
5.2 \\
25.9 \\
100\end{array}$ & $\begin{array}{c}1.000^{\ddagger} \\
0.605^{\ddagger} \\
0.291^{\ddagger} \\
0.549^{\star *} \\
0.045^{\star *} \\
<0.001^{\star *}\end{array}$ \\
\hline & \multicolumn{2}{|c|}{ Mediana (RIQ) } & \multicolumn{2}{|c|}{ Mediana (RIQ) } & \multicolumn{2}{|c|}{ Mediana (RIQ) } & \\
\hline Edad en años & \multicolumn{2}{|c|}{$56(49-67)$} & \multicolumn{2}{|c|}{$52(47-62)$} & \multicolumn{2}{|c|}{$60(49-69)$} & $0.018^{\dagger}$ \\
\hline $\begin{array}{l}\text { Características al ingreso a UCl } \\
\text { SAPS-3 (puntuación) } \\
\text { SOFA (puntuación) } \\
\text { Proteína C reactiva (mg/L) } \\
\text { Ferritina }(\mathrm{ng} / \mathrm{mL}) \\
\text { Dímero D }(\mu \mathrm{g} / \mathrm{L})\end{array}$ & \multicolumn{2}{|c|}{$\begin{array}{c}63(58-71) \\
11(7-12) \\
163(91-239) \\
802(478-1,382) \\
1,718(929-4,856)\end{array}$} & \multicolumn{2}{|c|}{$\begin{array}{c}60(55-64) \\
6(5-8) \\
117(54-206) \\
631(284-1,170) \\
1,250(781-2,556)\end{array}$} & \multicolumn{2}{|c|}{$\begin{array}{c}66(60-74) \\
11(11-12) \\
175(116-250) \\
914(543-1,500) \\
1,956(948-5,531)\end{array}$} & $\begin{array}{c}0.001^{\dagger} \\
<0.001^{\dagger} \\
0.048^{\dagger} \\
0.071^{\dagger} \\
0.123^{\dagger}\end{array}$ \\
\hline $\begin{array}{l}\text { Resultados } \\
\text { Días de ventilación mecánica } \\
\text { Días de estancia en UCl } \\
\text { Días de estancia en hospital }\end{array}$ & \multicolumn{2}{|c|}{$\begin{array}{c}8.5(4.8-14.9) \\
8.9(5.1-15.6) \\
15.5(8.5-23.3)\end{array}$} & \multicolumn{2}{|c|}{$\begin{array}{c}9.5(5.3-16.1) \\
11.5(6.8-18.2) \\
24.5(20.5-34.0)\end{array}$} & \multicolumn{2}{|c|}{$\begin{array}{c}8.0(4.1-15.3) \\
7.8(4.7-15.3) \\
10.8(7.6-17.7)\end{array}$} & $\begin{array}{c}0.357^{\dagger} \\
0.048^{\dagger} \\
<0.001^{\dagger}\end{array}$ \\
\hline
\end{tabular}

predominantemente por enfermedades respiratorias. La principal limitación de este estudio consistió en que se trató de la experiencia de una $\mathrm{UCl}$ en un solo centro de referencia.

\section{Conclusiones}

Este estudio representa la primera descripción de pacientes con ventilación mecánica infectados de SARS-CoV-2 en la población mexicana, que agrega información a la literatura acerca de la presentación y resultados tempranos. Datos epidemiológicos futuros determinarán si los hallazgos se correlacionan con otras regiones de México y Latinoamérica.

\section{Conflictos de intereses}

Los autores declaran no tener conflicto de intereses alguno.

\section{Financiación}

Los autores no recibieron patrocinio para llevar a cabo este artículo. 


\section{Responsabilidades éticas}

Protección de personas y animales. Los autores declaran que para esta investigación no realizaron experimentos en seres humanos ni en animales.

Confidencialidad de los datos. Los autores declaran que en este artículo no aparecen datos de pacientes.

Derecho a la privacidad y consentimiento infor-

mado. Los autores declaran que en este artículo no aparecen datos de pacientes.

\section{Bibliografía}

1. Mahase E. Covid-19: deaths in Mexico triple since reopening began in June. BMJ. 2020;370:m2753.

2. Auld SC, Caridi-Scheible M, Blum JM, Robichaux C, Kraft C, Jacob JT, et al. ICU and ventilator mortality among critically ill adults with coronavirus disease 2019. Crit Care Med. 2020;48:e799-e804.

3. Richardson S, Hirsch JS, Narasimhan M, Crawford JM, McGinn T, Davidson $\mathrm{KW}$, et al. Presenting characteristics, comorbidities, and outcomes among 5700 patients hospitalized with COVID-19 in the New York city area. JAMA. 2020;323:2052-2059.
4. Zhou F, Yu T, Du R, Fan G, Liu Y, Liu Z, et al. Clinical course and risk factors for mortality of adult inpatients with COVID-19 in Wuhan, China: a retrospective cohort study. Lancet. 2020;395:1054-1062.

5. Suárez V, Suárez-Quezada M, Oros-Ruiz S, Ronquillo-de Jesús E. Epidemiology of COVID-19 in Mexico: from the $27^{\text {th }}$ of February to the $30^{\text {th }}$ of April 2020. Rev Clin Esp. 2020;220:463-471.

6. Arentz M, Yim E, Klaff L, Lokhandwala S, Riedo FX, Chong M, et al. Characteristics and outcomes of 21 critically ill patients with COVID-19 in Washington State. JAMA. 2020;323:1612-1614.

7. Zangrillo A, Beretta L, Scandroglio AM, Monti G, Fominskiy E, Colombo S, et al. Characteristics, treatment, outcomes and cause of death of invasively ventilated patients with COVID-19 ARDS in Milan, Italy. Crit Care Resusc. 2020 Abr 23.

8. Ferrando C, Suárez-Sipmann F, Mellado-Artigas R, Hernández M, Gea A, Arruti E, et al. Clinical features, ventilatory management, and outcome of ARDS caused by COVID-19 are similar to other causes of ARDS. Intensive Care Med. 2020;46:2200-2211.

9. Kammar-García A, Vidal-Mayo JJ, Vera-Zertuche JM, Lazcano-Hernández M, Vera-López O, Segura-Badilla O, et al. Impact of comorbidities in Mexican sars-cov-2-positive patients: a retrospective analysis in a national cohort. Rev Invest Clin. 2020;72:151-158.

10. Ortiz-Brizuela E, Villanueva-Reza M, González-Lara MF, Tamez-Torres KM, Román-Montes CM, Díaz-Mejía BA, et al. Clinical and epidemiological characteristics of patients diagnosed with covid-19 in a tertiary care center in Mexico City: a prospective cohort study. Rev Invest Clin. 2020;72:165-177

11. Amit M, Sorkin A, Chen J, Cohen B, Karol D, Tsur AM, et al. Clinical course and outcomes of severe Covid-19: a national scale study. J Clin Med. 2020;9:2282. 